\title{
An android app for receiving, storing and exchanging glucose level data obtained from commercial glucometers
}

\begin{abstract}
This work shows the development of a device as an electronic interface and embedded software (EI), which obtains glucose level data from a commercial glucometer, sends this data to an application (APP) on an Android device. The APP stores the information in a database, shows various options for presenting the data and through short message messages (SMS) send the values of glucose levels and data of people who are in glucose control to an external repository, located in the Web cloud, where it is available to users and clinicians.
\end{abstract}

Keywords: glucose, device, glucometer, app, communication
Volume 5 Issue 5 - 2019

\author{
Jose de Jesus Sandoval-Palomares, Javier \\ Yañez-Mendiola \\ Department of Applied research,CIATEC, México
}

Correspondence: Jose de Jesus Sandoval-Palomares, Department ofApplied research,CIATEC,Omega 20I, Industrial Delta 37545, León Gto, México, Email jsando@ciatec.mx

Received: August 05, 2019 | Published: September27, 2019
Abbreviations: EI, electronic interface; APP, application; SMS, short message messages; API, application programming interface

\section{Introduction}

The use of telemedicine and digital tools interventions in diabetes care, seems to be a promising alternative to conventional therapy. ${ }^{1-4}$ Nowadays, mobile technologies are a very widespread and therefore viable alternative to help keep track of various medical control parameters..$^{4-8}$ Glucose level control helps reduce the incidence of longterm diabetic complicationsandthe continuous monitoring control of glucose levels is reflected in a decrease in levels and improvement indicators. ${ }^{9-12}$ Using the Internet or a phone where the glucosemonitoring data were transferred to web-based, and transmitted medical recommendations by short message, was as effective way for glucose control and other health behaviors. ${ }^{6,13,14}$ Diverse glucose reading devices have their own glucose level display mechanism, the traditional ones show in a monochromatic screen the numerical data of the reading made at that time or the last one made, date and time, some can show a limited history of numerical way; Onetouch, Contour TM, FreeStyle Lite, Accu-Chek, Bayer, to mention brands. An improvement like the FreeStyle Libre and Medisafe Fit Smile devices, they contain a screen that presents the glucose level currently read and a basic graph with previous hours and levels.

A substantial improvement of these devices is the use of wireless Bluetooth technology as the most widespread, which are communicated to smartphones or mobile device with IOS or Android operating system and a proprietary APP of the brand for glucose control; iHealth Wireless Smart Blood, OneTouch Verio Sync meter, FORA Test N'GO Voice Bluetooth are some with these features. The latest development is that the glucometer is connected directly to the phone or mobile device through a physical connection port; iHealth Align, Gmate SMART, Glucose, are some examples. On the other hand, there are currently APPs on the IOS and Android platform to monitor glucose levels and various diabetes control, mentioned in a long list to Diabetes Connect, BG Monitor Diabetes and Glucose Buddy.We found a tactic to impenetrate a mechanism, to obtain information directly from commercial glucometers through its physical connection using application programming interface (API), without having to use the proprietary APP of the brand, allowing to concentrate the information from multiple sources in a universal repository. Obtaining glucose results levels from different glucometers that have the possibility to send data physically or wirelessly is the objective of this project and implement a universal repository and not depend on brands to monitor and control the levels of glucose, covering an opportunity where users and clinicians do not have to be changing platforms, APP or repository to help with this problem.

\section{Methods}

The selected commercial glucometer is an ONETOUCH UltraMini, which can store several readings and has an API, which allows access to stored data. For programming software interfaces we used Android studio and SQLite for data storage. The APP has the ability to connect to more than one EI, since an individual who is controlling their glucose levels could have more than one glucometer in use.The general process that is carried out during the exchange of information between the EI, glucometer and APP is presented in detail in Figure 1.Figure 2 shows the process of receiving glucose level data and the processing carried out by the APP on Android and sending data via SMS. The different menu options that allow the user to interact with the APP are shown.

The APP was developed for Spanish language. It contains several menu options; Last Result (which shows the last data stored), Automatic Reception (which asks the data from the glucometer to the EI and this to the APP that automatically storage and send an SMS to externa Web-Database), Manual Send (which allows to manually capture a glucose level data), Query Results (allows visualization in the form of graph glucose data in a given period, day, week or month), Delete Results (which removes current glucose level data from the database), Visit Website (which sends to the http address where you 
can view the information sent to the Web-Database) and finally Exit (leaving the APP). The Figure 3 shows the menu options, that allow the user to interact with in the APP.

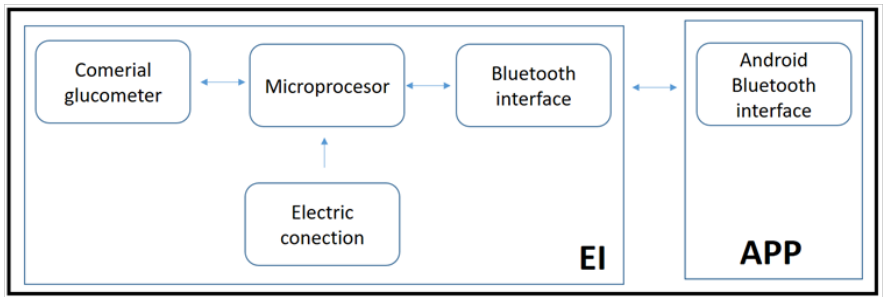

Figure I General process during the exchange of information between the (El) and glucometer.

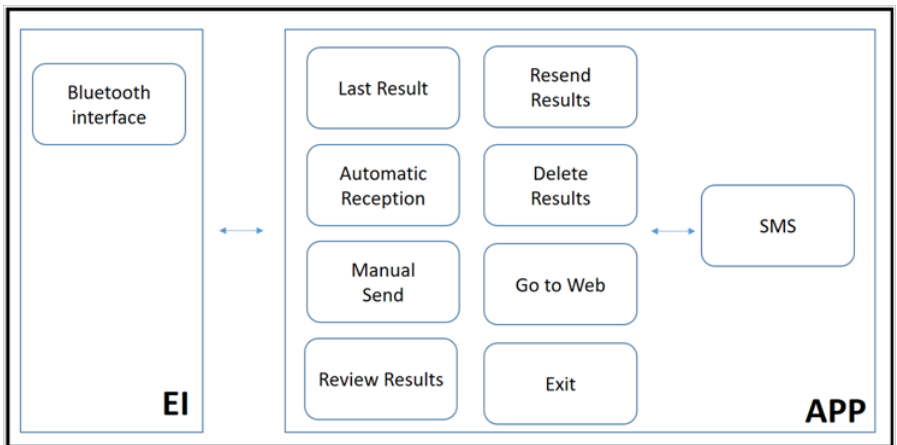

Figure 2 Process of receiving glucose level data and the processing carried out by the APP on Android.

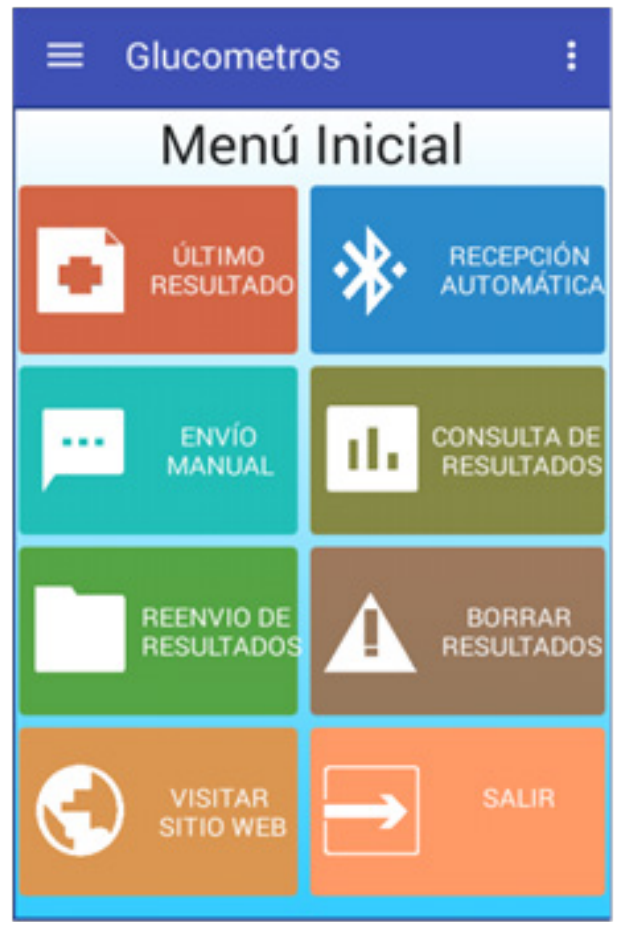

Figure 3 The menu options that allow the user to interact with in the APP.

\section{Processing}

When the App is initialized, it verified that the Bluetooth protocol is active on the mobile device and the permissions for connection are taken, If the connection is unsuccessful, after 5 attempts, the APP shows error message to verify: turned on the device's Bluetooth or turned on EI. When the connection is successful The EI requests the data from the glucometer and transforms it in a structure in format[IDGlucometer, Date, Time, GlucoseLevel], the APP receive this data frame, read it and sends a message to confirmation of the data reception. Received the information in the APP, the processing that separates the data is carried out, a verification is made that the data received is not repeated (previously stored), if this is the case it is discarded, in the case not repeated, a new one is made insertion into the local database and send a success message to the user.

\section{Final prototype}

A box was designed and created in 3D printing, to contain the EI system, including a 9v battery, an RGB LED was implemented to indicate the user when it is power on (blue), receiving data (blink of blue) or a problem was detected (red blink). This same box contains a compartment to place the commercial glucometer to facilitate interaction with the user. The Final prototype electronic interface (EI) and application (APP) on an Android device is shows in Figure 4.
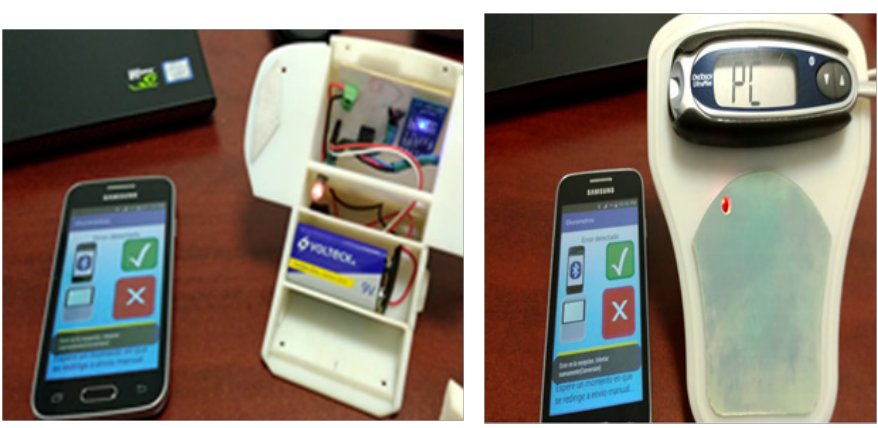

Figure 4 Final prototype electronic interface (EI) and application (APP) on an Android device.

\section{Testing process}

To verify the correct functionality of the system, different pilot tests were run using the EI and the APP. The following tests were executed 50 times; The IE was turned on and off, to verify that it is switched on and off correctly and the LED shows the correct blue color in switched on. The EI was connected and disconnected to the glucometer port and verify that its shows the legend PC on its screen, which indicates that it is ready to transfer data or the LED has a blink red colordue to bad connection problems. The APP was executed and connection attempts were applied using Bluetooth between an Android 6.0 operating system phone, the EI being switchedoff, verifying that the APP showed correctly when the EI was not available, while the IE is turned on the APP is connected correctly and will show successful connection and ready to initiate data transfers. Within the APP the different functionalities were tested, for this a document was followed based on use case and user case, which allowed verifying that the APP complied with each of the functionalities designed and encoded, in particular the functionality of automatic reception that is the one that interconnects all the elements was thoroughly tested, verifying that they correctly received the data frame of glucose level values, storage in the database of the Android device and sent by SMS of the data received to external Web-Database. The tests were performed by 3 users who are currently in control of their glucose levels, helping to identify recommendations on their use and functionality.

\section{Results}

The system as a whole performed satisfactorily. In the EI several 
tests were sent for sending data; containing information a single record in the glucometer, several records and even none. The LED color was presented according to the states implemented and the communication with the APP showed an acceptable performance by sending up to 100 stored records, which had not been previously sent. The EI was kept on for $4 \mathrm{hrs,} \mathrm{of} \mathrm{which} \mathrm{tests} \mathrm{were} \mathrm{sent} \mathrm{to} \mathrm{send} \mathrm{data}$ for 15 min intervals for every hour. The communication between the EI and APP components, via Bluetooth that was carried out in several states (with registers, without registers, several registers) presents a very fast response, without problems of sending or conversion of frame. The sending of SMS was successful and the reception by the receiving external Web-Database was completed smoothly.

Test users commented; They did not find it difficult to understand the operation of the system, the switching on and off of the EI made without difficulty, the LED helps to understand the stages of the process and it is useful to have more than one associated glucometer, due to loss, breakdown or change of the glucometer, even for travel situations, The APP is intuitive, useful and simple to use and sent via SMS is done without problem to the Web environment.

\section{Conclusion}

This document presents detail of the interconnection architecture of five elements that are currently clearly separated; glucometers, APP, Web-Database, patient users and medical specialists. The novelty of our proposal is that a proprietary APP of the glucometer manufacturer is not required, to obtain glucose level data, also, the current proprietary APPs only contains the functionality to visualize the data obtained from of their own glucometers. Another comparative novelty with existing non-proprietary APPs, ours obtains direct data from the glucometer, avoiding errors that users could have when entering their data, however we consider a manual capture useful and we implement this and complete the options of available capture. Other important novelty is the sending of glucose levels data by SMS to an external Web-Database, this form of sending does not depend on an internet connection, so that data could even be sent from remote places without an internet connection, connection that is indispensable in the other proprietaryand non-proprietary APPs. Some changes are necessary, such as implementing a warning in the EI when the battery is close to finish the charge or the possibility of a direct connection to the electricity socket. The APP only worked on version Android 6.0 operative system, which helped a lot to make it stable, but it reduces compatibility with current versions, so generating a more updated version might be required. Another 2 glucometers have already been identified and are working on making the connection with their APIs, expanding the range to use different brands and types of glucometers. This project opens the panorama, as it does not depend solely on the applications that commercial systems can offer, in addition to the fact that the type of glucometer used does not matter, clinicians and users may have only one interface to control glucose levels.

\section{Funding details}

Consejo Nacional de Ciencia y Tecnologia, México (National
Council for Science and Technology, CONACYT) andTecnoGadget, S. A de C.V. Mexican company.

\section{Acknowledgements}

None.

\section{Conflict of interests}

Authors declare that there is no conflict of interest.

\section{References}

1. Nishan Siriwardena LSA, Sudarshana Wickramasinghe WA, Dussantha Perera KL. A review of telemedicine interventions in diabetes care. $J$ Telemed Telecare. 2012;18(3):164-168.

2. Dy Aungst T, Ming Wai Tam L, Patel D. Digital health and the future of diabetes management. Journal of Diabetes, Metabolic Disorders \& Control. 2015;2(4):131-133.

3. Toledo FG, Triola A, Ruppert $\mathrm{K}$, et al. Telemedicine Consultations: An Alternative Model to Increase Access to Diabetes Specialist Care in Underserved Rural Communities. JMIR Res Protoc. 2012;1(2):e14.

4. Greenwood DA, Young HM, Quinn CC. Telehealth Remote Monitoring Systematic Review: Structured Self-monitoring of Blood Glucose and Impact on A1C. J Diabetes Sci Technol. 2014;8(2):378-389.

5. Istepanian RSH, Zitouni K, Harry D. Evaluation of a mobile phone telemonitoring system for glycaemic control in patients with diabetes. Journal of Telemedicine and Telecare. 2009:15(3):125-128.

6. Jae-Hyoung C, Hye-Chung L, Dong-Jun L. Mobile communication using a mobile phone with a glucometer for glucose control in Type 2 patient with diabetes: as effective as an Internet-based glucose monitoring system. Journal of Telemedicine and Telecare. 2009:15(2):77-82.

7. Anuradha Soni, Sandeep Kumar Jha. Smartphone based non-invasive salivary glucose biosensor. Analytica Chimica Acta. 2017; 996:54-63.

8. Szymborska-Kajaneka A, Psureka A, Heseb R, et al. Self-monitoring of blood glucose in treatment of type 2 diabetes. Diabetes research and clinical practice. 2009:49-52.

9. Litwak LE, Querzoli I, Musso C, et al. Continuous glucose monitoring. Utility and indications. Medicina (B Aires). 2019;79:44-52.

10. David C Klonoff . Continuous Glucose Monitoring: Roadmap for 21st century diabetes therapy. Diabetes Care. 2005:28(5):1231-1239.

11. Bode BW. Clinical Utility of the Continuous Glucose Monitoring System. Diabetes Technology \& Therapentics. 2004:2.

12. Bode BW, Gross TM, Thornton KR, et al. Continuous glucose monitoring used to adjust diabetes therapy improves glycosylated hemoglobin: a pilot study. Diabetes Research and Clinical Practice. 2000:47(3):225.

13. Kashem A, Droogan MT, Santamore WP, et al. Web-Based Internet Telemedicine Management of Patients with Heart Failure. Telemedicine and e-Health. 2006:12(4):439-447.

14. Fjeldsoe BS, Marshall AL, Miller YD. Behavior Change Interventions Delivered by Mobile Telephone Short-Message Service. American Journal of Preventive Medicine. 2009:36(2):165-173. 